C2019 IEEE. Personal use of this material is permitted. Permission from IEEE must be obtained for all other uses, in any current or future media, including reprinting/republishing this material for advertising or promotional purposes, creating new collective works, for resale or redistribution to servers or lists, or reuse of any copyrighted component of this work in other works. This is the author's version of an article that has been published in the conference proceedings. The final version of record is available at https://doi.org/10.1109/VTCFall.2019.8891511 


\title{
Data Association among Physical and Virtual Radio Transmitters with Visibility Regions
}

\author{
Markus Ulmschneider, Christian Gentner and Armin Dammann \\ German Aerospace Center (DLR), Institute of Communications and Navigation \\ Muenchner Str. 20, 82334 Wessling, Germany \\ \{markus.ulmschneider,christian.gentner,armin.dammann\}@dlr.de
}

\begin{abstract}
In multipath assisted positioning, multipath components (MPCs) are exploited for positioning as they are regarded as line-of-sight (LoS) signals from virtual transmitters. With simultaneous localization and mapping (SLAM), the locations of physical and virtual transmitters are estimated jointly with and relative to the user position. A robust data association scheme is crucial for the robustness of SLAM. In multipath assisted positioning, data association refers to the question which MPCs correspond to which transmitters. We say that a physical or virtual transmitter is visible to the user if the user is in LoS to the transmitter. Within this paper, we propose to map information on the visibility of physical and virtual transmitters in addition to their locations, and use such information for a reliable data association. Visibility information may stem from previous observations of a user, or from a visibility map of the scenario obtained from another user or a central entity. Our simulations in an indoor scenario show that information on the visibility of transmitters considerably improves the positioning performance by increasing the robustness of data association.

Index Terms-Channel-SLAM, data association, simultaneous localization and mapping
\end{abstract}

\section{INTRODUCTION}

Global navigation satellite systems (GNSSs) are able to satisfy the positioning performance needs of many services in areas with a clear view to the sky. In GNSS-denied scenarios like indoors or in urban canyons, the positioning performance may drastically shrink due to signal blocking and multipath propagation, for example. In many such scenarios, no positioning solution may be obtained at all with GNSSs [1].

Terrestrial signals like wireless local area network (WLAN) or cellular signals tend to have a much better coverage in these scenarios, and they can be used as signals of opportunity (SoOs) for positioning purposes [2]. However, multipath propagation still deteriorates the performance of systems based on such signals. The standard approach to cope with multipath propagation is to try to mitigate the influence of multipath components (MPCs) on the line-of-sight (LoS) path [3].

The idea of multipath assisted positioning is contrary to such approaches. Instead of regarding multipath propagation as an ill, the spatial information contained in MPCs is exploited for positioning. Each MPC arriving at a user can be treated as a signal from a virtual transmitter in a LoS condition. If the location of the physical transmitter and the environment of the scenario are known, for example by a floor plan, the locations of the virtual transmitters can be calculated in advance [4], [5].

However, neither the environment nor the location of the physical transmitter is known in the general case where a user enters an unknown scenario. In this case, the locations of the physical and virtual transmitters can be estimated jointly with the user position with simultaneous localization and mapping (SLAM) [6]-[12]. Therefore, depending on the scenario, positioning may be possible with only a single physical transmitter.

The authors of [6] have introduced such an approach named Channel-SLAM for terrestrial signals, which works in two stages. In the first stage, a channel estimator estimates the parameters of the signal components arriving at a receiver. In the second step, the locations of the physical and virtual transmitters and the position of the user are estimated with SLAM using recursive Bayesian estimation [13]. Thus, mapping in Channel-SLAM refers to estimating the locations of transmitters. Channel-SLAM does not differentiate between the LoS path and MPCs. Accordingly, there is no differentiation between physical and virtual transmitters.

A robust data association scheme is crucial for long-term SLAM. Data association tries to answer the question which measurements belong to which transmitters [14]. In [15], [16], a multiple hypothesis data association scheme based on a method from [17] has been introduced for Channel-SLAM.

When multiple users move in the same scenario, for example in museums, malls or public buildings, maps of estimated transmitter locations can be exchanged among users [18]. Information on the locations of transmitters obtained in form of a prior map may increase the positioning performance and decrease the convergence time in SLAM.

In addition to the transmitter locations, visibility regions of transmitters can be stored in a map. A visibility region describes the area with a LoS condition to a physical or virtual transmitter. Whether a virtual transmitter is in LoS at a specific location depends on the location of the physical transmitter and the scenario. Within this paper, we incorporate visibility regions into our multiple hypothesis data association scheme for Channel-SLAM. Additional information on visibility regions can on the one hand increase the robustness of the data association scheme, and on the other hand decrease the computational complexity. We expect that the positioning performance increases considerably if information on visibilities 


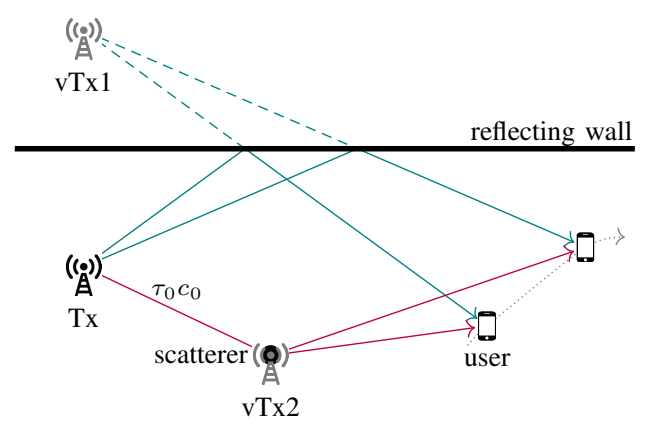

Fig. 1. The signal from the physical transmitter $\mathrm{Tx}$ is in the first case reflected at the wall. The corresponding MPC is treated as a LoS signal from the virtual transmitter vTx 1 , whose location is the location of Tx mirrored at the wall. In the second case, the MPC arriving at the user via the poin scatterer is treated as a LoS signal from the virtual transmitter vTx2, which is located at the scatterer location. The locations of both vTx 1 and vTx 2 are independent from the user position.

of transmitters from a prior map is incorporated into the data association process

The remainder of this paper is organized as follows. Section II introduces the idea of multipath assisted positioning and Channel-SLAM. In Section III] we introduce visibility regions and how they are incorporated into the data association scheme. Simulation results are presented in Section IV Section $\mathrm{V}$ concludes the paper.

\section{Multipath Assisted Positioning}

\section{A. Virtual Transmitters}

The idea of multipath assisted positioning is to regard MPCs as LoS signals from virtual transmitters. This idea is visualized in Fig. 1] The physical transmitter Tx broadcasts the transmit signal. Neglecting the LoS path, the user receives the signal via two different propagation paths at two different locations.

First, the signal is reflected at the wall. The reflected signal component arriving at the user can be regarded as a LoS signal from the virtual transmitter vTx 1 . Given the true time of flight and incoming angle of the signal component at the user, the location of vTx1 is the location of the physical transmitter mirrored at the surface. In case of a reflected signal, the physical and the virtual transmitter are perfectly time synchronized.

Second, the signal arrives at the point scatterer, which distributes the signal energy uniformly in all directions. The signal thus arrives again at the user as a MPC, which is regarded as a LoS component from the virtual transmitter vTx2. The location of vTx 2 coincides with the location of the point scatterer. In contrast to the case where the signal is reflected, the physical and the virtual transmitter are not synchronized in the case of a scattered signal component. There is an additional propagation delay $\tau_{0}$ between the two, which is their Euclidean distance divided by the speed of light $c_{0}$. The propagation delay can be regarded as a clock offset, or an additional propagation distance.

In both of the above cases, the location of the virtual transmitter does not depend on the position of the user, but it is static. Furthermore, the two cases can be generalized to the case where a signal is reflected and/or scattered multiple times on the way from the physical transmitter to the user [6].

\section{B. Channel-SLAM}

The propagation channel we consider is a time-variant linear multipath channel [19]. Therefore, the signal transmitted by a physical transmitter arrives at the user via different propagation paths. At the receiver, a linear superposition of signal components is received. The signal components are distinguished by their time of arrival (ToA), angle of arrival (AoA), received signal strength and phase, for example.

The Channel-SLAM algorithm works in two steps. In the first step, after sampling the received signal at the user side, the parameters of the signal components are estimated by a channel estimator. We use the Kalman enhanced super resolution tracking (KEST) estimator [20], which works in two stages. The inner stage estimates the signal components' parameters snapshot-wise based on the estimated channel impulse response (CIR). For this estimation, we use the Space-Alternating Generalized Expectation-Maximization (SAGE) algorithm [21], which is a variant of the ExpectationMaximization (EM) [22] algorithm. The outer stage of KEST tracks the estimates from the inner stage over time using Kalman filters. This outer stage inherently tackles the problem of associating signal components from one time instant to another. In addition, the number of signal components is tracked in the outer stage. Within the scope of this paper, we use only the ToA and AoA estimates from the channel estimator. Thus, the ToA estimates for the $N_{\text {TX }}$ transmitters at time instant $k$ are stacked in the vector

$$
\boldsymbol{d}_{k}=\left[\begin{array}{lll}
d_{1, k} & \ldots & d_{N_{\mathrm{TX}}, k}
\end{array}\right]^{T} .
$$

Note that the number of transmitters may change over time depending on the position of the user. Nevertheless, the time index $k$ is omitted in $N_{\mathrm{TX}}$ for notational convenience. We assume that the user is equipped with an antenna array that is coupled with the user's orientation. The AoA estimates at time instant $k$ are stacked in the vector

$$
\boldsymbol{\theta}_{k}=\left[\begin{array}{lll}
\theta_{1, k} & \ldots & \theta_{N_{\mathrm{TX}}, k}
\end{array}\right]^{T} .
$$

In the second step of Channel-SLAM, the position and the velocity of the user and the locations and clock offsets of the transmitters are estimated jointly using SLAM. As each signal component corresponds to one transmitter, the estimates from the channel estimator serve as measurement input in SLAM. The measurement vector at time instant $k$ is therefore

$$
\boldsymbol{z}_{k}=\left[\begin{array}{ll}
\boldsymbol{d}_{k}^{T} & \boldsymbol{\theta}_{k}^{T}
\end{array}\right]^{T}
$$

Because the transmitters are static in our model, their state comprises their location in two dimensions and a clock offset $\tau_{0}$. Thus, the state vector for the $j^{\text {th }}$ transmitter at time instant $k$ is given by

$$
\boldsymbol{x}_{\mathrm{TX}, k}^{<j>}=\left[\begin{array}{lll}
x_{\mathrm{TX}, k}^{<j>} & y_{\mathrm{TX}, k}^{<j>} & \tau_{0, k}^{<j>}
\end{array}\right]^{T} .
$$


The state of the user is their position and velocity in two dimensions each. The user state vector at time instant $k$ is

$$
\boldsymbol{x}_{\mathrm{u}, k}=\left[\begin{array}{llll}
x_{k} & y_{k} & v_{x, k} & v_{y, k}
\end{array}\right]^{T} .
$$

The entire state vector at time instant $k$ is hence

$$
\begin{aligned}
\boldsymbol{x}_{k} & =\left[\begin{array}{llll}
\boldsymbol{x}_{\mathrm{u}, k}^{T} & \boldsymbol{x}_{\mathrm{TX}, k}^{T}
\end{array}\right]^{T} \\
& =\left[\begin{array}{llll}
\boldsymbol{x}_{\mathrm{u}, k}{ }^{T} & \boldsymbol{x}_{\mathrm{TX}, k}^{<1>T} & \ldots & \boldsymbol{x}_{\mathrm{TX}, k}^{<N_{\mathrm{TX}}>T}
\end{array}\right]^{T} .
\end{aligned}
$$

We are interested in the minimum mean square error (MMSE) estimator for the state from time instants zero to $k$, $\boldsymbol{x}_{0: k}$. With $\boldsymbol{z}_{1: k}$ denoting the measurements from time instants 1 to $k$, the estimator is

$$
\hat{\boldsymbol{x}}_{0: k}=\int \boldsymbol{x}_{0: k} \mathrm{p}\left(\boldsymbol{x}_{0: k} \mid \boldsymbol{z}_{1: k}\right) \mathrm{d} \boldsymbol{x}_{0: k} .
$$

In order to obtain the posterior probability density function (PDF) $\mathrm{p}\left(\boldsymbol{x}_{0: k} \mid \boldsymbol{z}_{1: k}\right)$, we use Bayesian recursive estimation [13]. The posterior PDF is calculated recursively. With the control input $\boldsymbol{u}$, which may be obtained from an inertial measurement unit (IMU), for example, it can be factorized as

$$
\begin{aligned}
\mathrm{p}\left(\boldsymbol{x}_{0: k} \mid \boldsymbol{z}_{1: k}, \boldsymbol{u}_{1: k}\right)= & \mathrm{p}\left(\boldsymbol{x}_{\mathrm{TX}, 0: k}, \boldsymbol{x}_{\mathrm{u}, 0: k} \mid \boldsymbol{z}_{1: k}, \boldsymbol{u}_{1: k}\right) \\
= & \mathrm{p}\left(\boldsymbol{x}_{\mathrm{u}, 0: k} \mid \boldsymbol{z}_{1: k}, \boldsymbol{u}_{1: k}\right) \\
& \times \mathrm{p}\left(\boldsymbol{x}_{\mathrm{TX}, 0: k} \mid \boldsymbol{z}_{1: k}, \boldsymbol{x}_{\mathrm{u}, 0: k}\right) \\
= & \mathrm{p}\left(\boldsymbol{x}_{\mathrm{u}, 0: k} \mid \boldsymbol{z}_{1: k}, \boldsymbol{u}_{1: k}\right) \\
& \times \prod_{j=1}^{N_{\mathrm{TX}}} \mathrm{p}\left(\boldsymbol{x}_{\mathrm{TX}, 0: k}^{<j>} \mid \boldsymbol{x}_{\mathrm{u}, 0: k}, \boldsymbol{z}_{1: k}\right) .
\end{aligned}
$$

In the last step of Eq. (7), we assume independence among the estimates for the single signal components in the channel estimator. This implies independence among measurements for the single transmitters and allows to estimate the states of the transmitters independently from each other. We use a RaoBlackwellized particle filter [23] to solve the SLAM problem. The posterior PDF of the current user state is thus expressed as a sum of user particles, where the $i^{\text {th }}$ particle is denoted by $\boldsymbol{x}_{\mathrm{u}, k}^{<i>}$ and has a weight $w_{k}^{<i>}$ associated to it. Therefore, we have

$$
\mathrm{p}\left(\boldsymbol{x}_{\mathrm{u}, k} \mid \boldsymbol{z}_{1: k}, \boldsymbol{u}_{1: k}\right)=\sum_{i=1}^{N_{p}} w_{k}^{<i>} \delta\left(\boldsymbol{x}_{\mathrm{u}, k}-\boldsymbol{x}_{\mathrm{u}, k}^{<i>}\right),
$$

where $\delta(\cdot)$ is the Dirac delta function. The user state is estimated by a sequential importance resampling (SIR) particle filter [24]. Given the structure of the Rao-Blackwellized particle filter, the transmitters' state is estimated for each user particle independently from the other user particles. Assuming independence among the measurements for the transmitters, each of the $N_{\text {TX }}$ transmitter states is estimated by a particle filter for each of the user particles. The $\ell^{\text {th }}$ transmitter particle for the $j^{\text {th }}$ transmitter of the $i^{\text {th }}$ user particle is denoted by $\boldsymbol{x}_{\mathrm{TX}, k}^{<i, j, \ell>}$ and has a weight $w_{k}^{<i, j, \ell>}$ associated to it. Thus, the

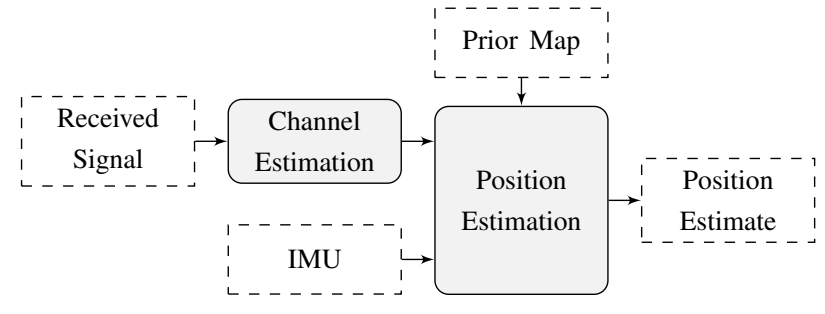

Fig. 2. Overview of Channel-SLAM. The channel estimator estimates the parameters of signal components in a first step. In the second step, these estimates are used as measurement input for SLAM to estimate the location of the transmitters and the position of the user. Additional sensors such as an IMU or prior information such as from a map may be fused in SLAM.

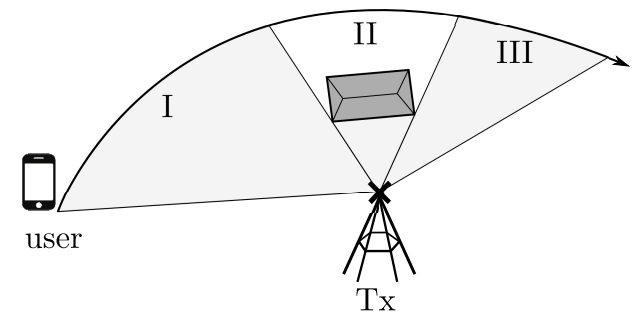

Fig. 3. The signal from the transmitter Tx can be received by the user in Region I. After the signal is blocked in Region II, it can be observed again by the user in Region III.

corresponding transmitter's posterior PDF is expressed as

$$
\begin{aligned}
\mathrm{p} & \left(\boldsymbol{x}_{\mathrm{TX}, k}^{<i, j>} \mid \boldsymbol{z}_{1: k}, \boldsymbol{x}_{\mathrm{u}, k}^{<i>}\right) \\
& =\sum_{\ell=1}^{N_{p, \mathrm{Tx}}} w_{k}^{<i, j, \ell>} \delta\left(\boldsymbol{x}_{\mathrm{TX}, k}^{<i, j>}-\boldsymbol{x}_{\mathrm{TX}, k}^{<i, j, \ell>}\right),
\end{aligned}
$$

where $N_{p, \text { Tx }}$ denotes the number of transmitter particles. Although $N_{p, \text { Tx }}$ may vary for different user particles, time instants and transmitters, we omit the corresponding indices for notational convenience. In order to decrease the complexity of Channel-SLAM, we apply a particle reduction method for the transmitter particle filters. The transmitter state space is divided into discrete bins, and in every bin only a number $N_{p, \text { Tx }}^{\mathrm{B}}$ of particles is allowed. For transmitters with a small uncertainty on their state, this method decreases the number of needed particles and thus the complexity of Channel-SLAM, while it hardly influences the positioning performance [25].

Fig. 2 gives an overview of the two steps in Channel-SLAM. In the first step, the parameters of the signal components are estimated based on the received signal by the channel estimator. In the second step, these estimates are used as measurements for the actual estimation of the states of the user and the transmitters. For this position estimation, additional sensors such as an IMU may be integrated. Furthermore, prior information such as from a transmitter map obtained from a central entity or other users can be exploited.

\section{DATA ASSOCIATION AND MAPPING}

In Channel-SLAM, the term mapping refers to the estimation of the states of the physical and virtual radio transmitters as in Eq. (4). In addition to these states, information on the 
locations with a LoS condition to a transmitter, i.e., where a transmitter is visible and where not, can be stored in a map. Fig. 3 illustrates such visibility regions. The user moves on its trajectory. In Region I, the signal from the transmitter Tx can be received, i.e., the transmitter is visible. In Region II, the signal from $\mathrm{Tx}$ to the user is blocked, while in Region III, the signal can be received again. Within this section, we expand Channel-SLAM by estimating information on visibility regions in a map and integrate it in our data association scheme proposed in [15]. Data association in Channel-SLAM refers to the question which measurement corresponds to which transmitter. For example, in Fig. 3, the user has to correctly assign the signal received in Region III to the transmitter Tx, whose signal was received already in Region I.

\section{A. Data Association in Channel-SLAM}

As the user moves through the scenario, the channel estimator may detect new signal components, i.e., new transmitters. When such a new signal component is detected, it may be associated with a transmitter that had been observed before.

We denote the set of transmitters that the user had observed before, but not any more at the current time instant $k$, by $\Upsilon_{k}$. The association variable $n_{k}$ denotes the association of the transmitter initialized at time instant $k$ with the transmitter $n_{k} \in \Upsilon_{k}$. In [15], we have presented a multiple hypothesis tracking data association scheme for Channel-SLAM based on the method in [17]. Each user particle takes association decisions on its own, and thus carries one hypothesis for the correct associations. A set of association decisions up to time instant $k-1$ for the $i^{\text {th }}$ user particle is stored in the variable $\hat{n}_{k-1}^{<i>}$.

Given the measurements $\boldsymbol{z}_{k}$ at the current time instant $k$, we can calculate the marginalized likelihood $p_{z, n_{k}}^{<i>}$ of the measurement for the $i^{\text {th }}$ particle assuming that the newly detected transmitter corresponds to the transmitter $n_{k} \in \Upsilon_{k}$. This likelihood is calculated as [15]

$$
\begin{aligned}
p_{z, n_{k}}^{<i>} & =\mathrm{p}\left(\boldsymbol{z}_{k} \mid n_{k}, \hat{n}_{k-1}^{<i>}, \boldsymbol{x}_{\mathrm{u}, k}^{<i>}, \boldsymbol{z}_{1: k-1}\right) \\
& =\sum_{\ell=1}^{N_{p, \mathrm{Tx}}} w_{k}^{<i, n_{k}, \ell>} \mathrm{p}\left(\boldsymbol{z}_{k} \mid \boldsymbol{x}_{\mathrm{TX}, k}^{<i, n_{k}, \ell>}, n_{k}, \hat{n}_{k-1}^{<i>}, \boldsymbol{x}_{\mathrm{u}, k}^{<i>}\right) .
\end{aligned}
$$

In addition to the above marginalized likelihoods $p_{z, n_{k}}^{<i>}$, we define a likelihood $p_{z, 0}^{<i>}$ which represents a likelihood that no association is made, i.e., that the newly detected transmitter is indeed a new transmitter that has not been observed by the user before. A hard association decision for the $i^{\text {th }}$ user particle is then sampled from the the likelihoods $p_{j}^{<i>}=p_{z, j}^{<i>}$, where $j \in\left\{0 \cup \Upsilon_{k}\right\}$. If no association is made, the new transmitter is initialized based on the corresponding measurement in $\boldsymbol{z}_{k}$. Otherwise, the transmitter can be initialized with the state estimate of the associated transmitter $n_{k}$, which is likely to have a smaller uncertainty than a transmitter that has to be initialized based on the measurement. Thus, with correct associations, we expect a shorter convergence time and a better positioning performance.

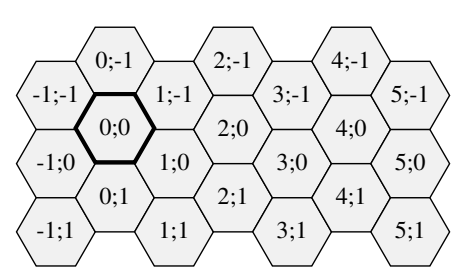

Fig. 4. Hexagonal grid to store information on visibility regions of transmitters in an occupancy grid map. Each hexagon is assigned a unique index.

Wrong association decisions can lead to a divergence of the filter. Particles with wrong association hypotheses will have a small likelihood as the user moves, and therefore their weights decrease. Such particles are unlikely to be resampled in the resampling step of the particle filter. Hence, the filter inherently eliminates wrong association decisions. However, association ambiguities due to the geometry of the scenario may lead to a deterioration of the performance of ChannelSLAM in terms of accuracy and complexity.

\section{B. Mapping of Visibility Regions of Transmitters}

In order to increase the robustness of our data association scheme described above, we include visibility regions of transmitters. Due to a possibly high uncertainty in a transmitter's state, for example due to a high geometrical dilution of precision (GDoP), we do not use a parametric approach based on the transmitter's location to describe visibility regions. Instead, we store visibility information in a location-based map [26]. In particular, we use a hexagonal grid as in Fig. 4

The probability that the $j^{\text {th }}$ transmitter is visible in hexagon $h$ is in general unknown. We denote the random variable for this probability by $\boldsymbol{V}_{h}^{<j>}$. The set of the random variables for the $N_{\mathrm{TX}}$ transmitters' visibilities in hexagon $h$ is denoted by $\boldsymbol{M}_{h}$, which is

$$
\boldsymbol{M}_{h}=\left\{\boldsymbol{V}_{h}^{<1>}, \ldots, \boldsymbol{V}_{h}^{<N_{\mathrm{TX}}>}\right\} .
$$

A hexagonal visibility map $M$ is defined by the set $\left\{\boldsymbol{M}_{1}, \ldots, \boldsymbol{M}_{N_{h}}\right\}$, where $N_{h}$ is the number of hexagons in the map. The probability where a transmitter is visible and where not needs to be estimated by a user traveling through the scenario. Therefore, Eq. (7) is expanded as

$$
\begin{aligned}
\mathrm{p} & \left(\boldsymbol{x}_{0: k}, \boldsymbol{M} \mid \boldsymbol{z}_{1: k}, \boldsymbol{u}_{1: k}\right) \\
& =\mathrm{p}\left(\boldsymbol{x}_{0: k} \mid \boldsymbol{z}_{1: k}, \boldsymbol{u}_{1: k}\right) \mathrm{p}\left(\boldsymbol{M} \mid \boldsymbol{x}_{0: k}, \boldsymbol{z}_{1: k}, \boldsymbol{u}_{1: k}\right) \\
& =\mathrm{p}\left(\boldsymbol{x}_{0: k} \mid \boldsymbol{z}_{1: k}, \boldsymbol{u}_{1: k}\right) \mathrm{p}\left(\boldsymbol{M} \mid \boldsymbol{x}_{0: k}, \boldsymbol{z}_{1: k}\right) \\
& =\mathrm{p}\left(\boldsymbol{x}_{0: k} \mid \boldsymbol{z}_{1: k}, \boldsymbol{u}_{1: k}\right) \prod_{h=1}^{N_{h}} \mathrm{p}\left(\boldsymbol{M}_{h} \mid \boldsymbol{x}_{0: k}, \boldsymbol{z}_{1: k}\right) \\
& =\mathrm{p}\left(\boldsymbol{x}_{0: k} \mid \boldsymbol{z}_{1: k}, \boldsymbol{u}_{1: k}\right) \prod_{h=1}^{N_{h}} \prod_{j=1}^{N_{\mathrm{TX}}} \mathrm{p}\left(\boldsymbol{V}_{h}^{<j>} \mid \boldsymbol{x}_{0: k}, \boldsymbol{z}_{1: k}\right) .
\end{aligned}
$$

The distribution of the visibility map $M$ in Eq. (13) is conditioned on both the user trajectory $\boldsymbol{x}_{0: k}$ and the history of measurements $\boldsymbol{z}_{1: k}$. The measurement history is only needed to indicate which transmitters have been visible at which 
time step, whereas the actually measured values are not used to estimate the visibility map. Following Eq. (12), the map estimation problem can be separated from Channel-SLAM. In addition, due to the hexagonal structure of the map and the independence among visibilities for single transmitters, it can be broken down to $N_{h} N_{\mathrm{TX}}$ subproblems as in Eq. (15). Thus, we map visibilities of each transmitter for each single hexagon.

Each user particle stores a list of hexagons that it has visited. For notational convenience though, we drop the user particle index $i$ in the variables introduced the following. In each hexagon, we store two counters for each transmitter in the scenario. For hexagon $h$ in the hexagon list of a user particle, the counter $V_{h, k}^{<j>}$ counts the number of times that a signal from transmitter $j$ has been received in this hexagon, i.e., that the $j^{\text {th }}$ transmitter was visible, between time instants zero and $k$. Likewise, the counter $\bar{V}_{h, k}^{<j>}$ counts the number of times that transmitter $j$ was not visible. The counters of hexagon $h$ are updated at time instant $k$ in one of the following two cases. In the first case, the user particle leaves a hexagon and enters hexagon $h$. If transmitter $j$ is visible, $V_{h, k}^{<j>}$ is increased by one, and otherwise $\bar{V}_{h, k}^{<j>}$ is increased by one. In the second case, the user stays in hexagon $h$ between time instants $k-1$ and $k$, but the visibility of one or more transmitters changes from not visible to visible or vice versa. If transmitter $j$ is such a transmitter, either $V_{h, k}^{<j>}$ or $\bar{V}_{h, k}^{<j>}$ is increased by one depending whether the transmitter is now visible or not.

As the user moves through the scenario, each user particle creates an estimate for the visibility map $\boldsymbol{M}$ as described above. We assume that $\mathrm{p}\left(\boldsymbol{V}_{h}^{<j>} \mid \boldsymbol{x}_{0: k}, \boldsymbol{z}_{1: k}\right)$ follows a Beta distribution with parameters $V_{h, k}^{<j>}+\nu_{h}^{<j>}$ and $\bar{V}_{h, k}^{<j>}+\bar{\nu}_{h}^{<j>}$, where $\nu_{h}^{<j>}$ and $\bar{\nu}_{h}^{<j>}$ are prior counts, which can be used to incorporate prior knowledge on visibility regions and to tune the trust into the user observations on visibilities. The PDF of a Beta distribution $\mathbb{B}(x ; p, q)$ with parameters $p$ and $q$ is defined by

$$
\mathbb{B}(x ; p, q)=\frac{1}{\mathrm{~B}(p, q)} x^{p}(1-x)^{q}
$$

for $x \in(0,1)$, and $\mathbb{B}(x ; p, q)=0$ otherwise. The Beta function normalizes the Beta distribution, and it is defined by

$$
\mathrm{B}(p, q)=\frac{\Gamma(p) \Gamma(q)}{\Gamma(p+q)},
$$

where $\Gamma(\cdot)$ is the Gamma function.

\section{Data Association with Visibility Regions}

For associating a newly detected signal component with a previously observed transmitter, we have two different sources of information. The first one is the actual measurement, i.e., ToA and AoA, for the signal component together with the location of the user particle and the estimates for previously observed transmitters' states. This information can be exploited as shown in Subsection III-A. The second one is the information on visibilities of transmitters in the hexagonal visibility map as in Subsection III-B together with corresponding

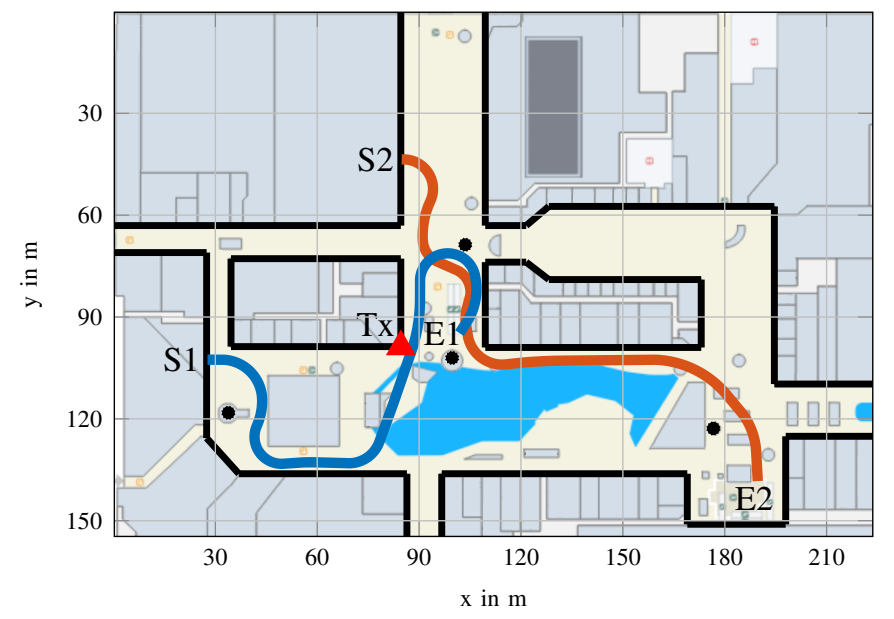

Fig. 5. Top view on the urban simulation scenario with one physical transmitter marked by the red triangle labeled Tx. The two user tracks start at $\mathrm{S} 1$ and $\mathrm{S} 2$ and end at E1 and E2, respectively. Bold black lines represent reflecting walls, whereas black dots represent point scatterers.

the user particle location. If a user comes back to a previously visited hexagon, the information in the hexagon on which transmitters were previously visible and which were not can be used.

To fuse both sources, the likelihoods $p_{z, n_{k}}^{<i>}$ in Eq. 10 are weighted by a factor $p_{h, n_{k}}^{<i>}$, which incorporates visibility information. We define $p_{h, n_{k}}^{<i>}$ as the expectation value of $\mathrm{p}\left(\boldsymbol{V}_{h}^{<n_{k}>} \mid \boldsymbol{x}_{0: k}, \boldsymbol{z}_{1: k}\right)$ for the $i^{\text {th }}$ user particle, which is

$$
\begin{aligned}
p_{h, n_{k}}^{<i>} & =\mathbb{E}\left[\mathrm{p}\left(\boldsymbol{V}_{h}^{<n_{k}>} \mid \boldsymbol{x}_{0: k}, \boldsymbol{z}_{1: k}\right)\right] \\
& =\frac{V_{h, k}^{<n_{k}>}+\nu_{h}^{<n_{k}>}}{V_{h, k}^{<n_{k}>}+\nu_{h}^{<n_{k}>}+\bar{V}_{h, k}^{<n_{k}>}+\bar{\nu}_{h}^{<n_{k}>} .}
\end{aligned}
$$

Finally, the $i^{\text {th }}$ particle samples its association decisions from

$$
p_{n_{k}}^{<i>}=p_{z, n_{k}}^{<i>} \times p_{h, n_{k}}^{<i>},
$$

where $n_{k} \in\left\{0 \cup \Upsilon_{k}\right\}$, and $p_{h, 0}^{<i>}=\frac{1}{2}$.

In the above method, we assume that at most one transmitter is initialized at every time step. In [16], we have proposed a method based on a greedy algorithm to drop this assumption. In addition, the paper shows how to incorporate the information on transmitters contained in prior maps obtained from different users, for example, in the data association scheme. It is straightforward to also adapt the data association algorithm with hexagonal visibility maps to the case where prior maps including visibility information are available.

\section{Simulations}

We evaluate our data association method by simulations in an indoor scenario. The top view of a mall with one physical transmitter labeled $\mathrm{Tx}$ and depicted by the red triangle is shown in Fig. 5. The physical transmitter continuously broadcasts a signal of $100 \mathrm{MHz}$ bandwidth which is known to the users. The signal is reflected by walls that are represented by the thick black lines, and scattered by point scatterers 
depicted by black dots. The users know the location of neither the physical transmitter nor the walls and scatterers. For the simulations, we incorporate reflection and scattering of orders one and two. Hence, we have single and double reflections and/or scattering. At each user position, the CIR is created by ray tracing. The received signal is created by limiting the CIRs to the $100 \mathrm{MHz}$ bandwidth and adding white Gaussian noise of constant power.

There are two different user tracks in the scenario. The $i^{\text {th }}$ user walks along their track from the starting point $\mathrm{S}_{\mathrm{i}}$ to the end point $E_{i}$ with a constant speed of $1 \mathrm{~m} / \mathrm{s}$. Both users record a snapshot of the received signal every $10 \mathrm{~ms}$. They are equipped with antenna arrays consisting of nine elements. With the KEST estimator, the ToAs and AoAs of each detected signal component are estimated in each snapshot. In addition, we assume that each user carries an IMU with them. From the IMU, however, we integrate only the heading information from the gyroscope into our movement model and do not use acceleration measurements.

The starting positions and headings of the users are assumed to be known in the simulations. The user particle filter uses 1000 particles, whereas the number of particles for the transmitters is adapted to the current certainty on the transmitter's state. A transmitter with a lower uncertainty on its state can be represented with a smaller number of particles. Thus, the number of particles of the transmitters differs depending on the corresponding user particles, transmitters and time instants.

We assume the users have obtained a prior map of transmitters of the scenario from one or more other users or a central entity. The map consists of two parts. The first part is an estimate of transmitter states, i.e., locations and clock offsets. We fill the map with the physical transmitter and the virtual transmitters whose states are calculated based on the floor plan of the scenario. We include virtual transmitters up to an order of two, i.e., single and double reflections and/or scattering. The transmitter state PDFs in the map are unbiased towards the true transmitter locations and follow a Gaussian distribution with a variance of $3 \mathrm{~m}^{2}$ in each spatial dimension, and a variance of $1 \mathrm{~m}^{2}$ in their additional propagation distance. The second part is a hexagonal map on transmitter visibility areas. For each hexagon $h$, the prior counts $\nu_{h}$ and $\bar{\nu}_{h}$ in Eq. (19) are set as follows. If a transmitter is visible from the center of the hexagon, $\nu_{h}=1+\epsilon_{\nu}$ and $\bar{\nu}_{h}=\bar{\epsilon}_{\nu}$, where $\epsilon_{\nu}=0.01$. Otherwise, $\nu_{h}=\epsilon_{\nu}$ and $\bar{\nu}_{h}=1+\bar{\epsilon}_{\nu}$. The side length of the hexagons is $2 \mathrm{~m}$.

Two cases are compared for each user in the following In both cases, newly detected transmitters may be associated with transmitters from the prior map or previously observed transmitters. In the first case, the user applies the data association scheme from [16], which is summarized in Subsection III-A and does not incorporate any information on transmitter visibility areas. It solely relies on the information on transmitter locations. This case will be referred to as plain $D A$. In the second case, the information on visibility areas is incorporated in addition into the estimation process as explained in Subsection III-C. This case will be referred to
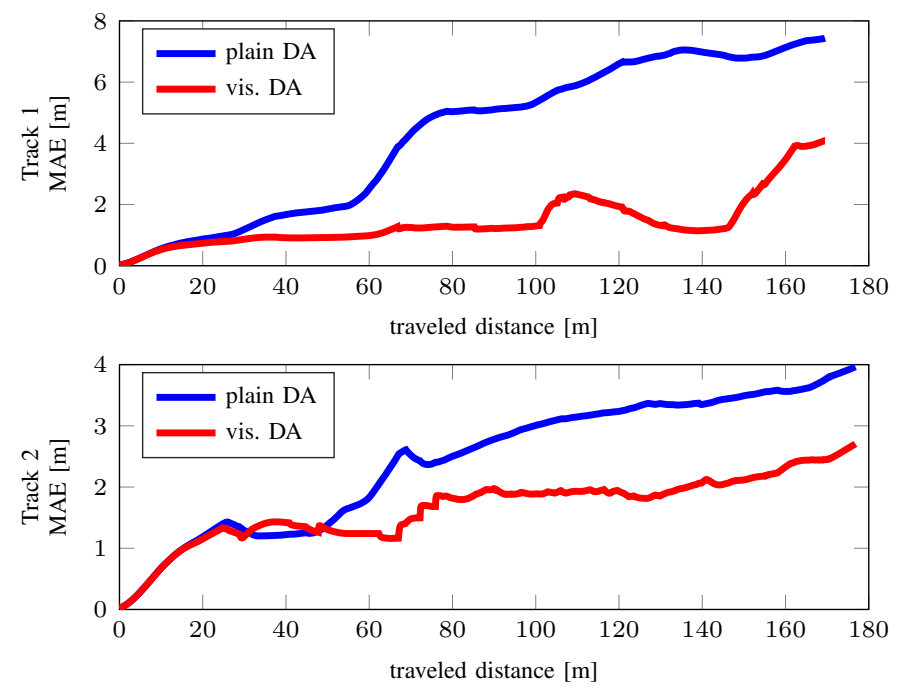

Fig. 6. The MAE of the estimated locations of the two users versus the traveled distance. The blue curve is the MAE if no visibility information is used for data association (plain DA), while for the red curve the visibility regions for transmitters are incorporated (vis. DA).

as vis. $D A$.

The results of the simulations in terms of positioning performance are shown in Fig. 6. The results are averaged over 200 runs. The mean absolute errors (MAEs) of the user positions are plotted against the traveled distance in blue if no visibility information is used from the prior map (plain DA), and in red if this information is exploited (vis. DA). As the starting locations and headings of the users are known, the initial MAE is zero. In the beginning of the tracks, the positioning performances are equal in both cases. As the user starts to move, associations of newly detected signal components can be made with transmitters from the prior map. We observe that then the MAE is in the most cases significantly smaller if visibility information is incorporated.

\section{CONCLusion}

A reliable data association scheme is crucial for long term robust SLAM. Within this paper, we have extended our previous data association scheme for Channel-SLAM by integrating visibility information of transmitters. When a user walks through the scenario, they collect information on the visibilities of transmitters, and use such information for data association. In addition, maps of transmitter locations may be shared among users. We have shown by simulations that augmenting such maps by transmitter visibility information improves the robustness of our data association scheme and thus the positioning performance of a user receiving a prior map.

\section{ACKNOWLEDGEMENT}

This work was partially supported by the the DLR project Navigation 4.0. 


\section{REFERENCES}

[1] P. Misra and P. Enge, Global Positioning System: Signals, Measurements, and Performance. Ganga-Jamuna Press, 2011.

[2] P. Dabove, A. Andrea, and V. D. Pietra, "Positioning Techniques with Smartphone Technology: Performances and Methodologies in Outdoor and Indoor Scenarios," in Smartphones from an Applied Research Perspective, N. Mohamudally, Ed. Rijeka: InTech, 2017, ch. 8.

[3] M. Lentmaier and B. Krach, "Maximum Likelihood Multipath Estimation in Comparison with Conventional Delay Lock Loops," in Proceedings of the 19th International Technical Meeting of the Satellite Division of The Institute of Navigation (ION GNSS), Sep. 2006.

[4] P. Meissner, C. Steiner, and K. Witrisal, "UWB positioning with virtual anchors and floor plan information," in Positioning Navigation and Communication (WPNC), 2010 7th Workshop on, March 2010, pp. 150156.

[5] P. Setlur, G. Smith, F. Ahmad, and M. Amin, "Target Localization with a Single Sensor via Multipath Exploitation," IEEE Trans. Aerosp. Electron. Syst., vol. 48, no. 3, pp. 1996-2014, Jul. 2012

[6] C. Gentner, T. Jost, W. Wang, S. Zhang, A. Dammann, and U.-C. Fiebig, "Multipath Assisted Positioning with Simultaneous Localization and Mapping," IEEE Trans. Wireless Commun., vol. 15, no. 9, pp. 6104 6117, Sep. 2016

[7] K. Witrisal, P. Meissner, E. Leitinger, Y. Shen, C. Gustafson, F. Tufvesson, K. Haneda, D. Dardari, A. F. Molisch, A. Conti, and M. Z. Win, "High-Accuracy Localization for Assisted Living - 5G Systems will turn Multipath Channels from Foe to Friend," IEEE Signal Process. Mag., vol. 33, no. 2, pp. 59-70, Mar. 2016.

[8] R. Mendrzik, H. Wymeersch, G. Bauch, and Z. Abu-Shaban, "Harnessing NLOS Components for Position and Orientation Estimation in 5G Millimeter Wave MIMO," IEEE Trans. Wireless Commun., vol. 18, no. 1, pp. 93-107, Jan. 2019.

[9] E. Leitinger, F. Meyer, F. Tufvesson, and K. Witrisal, "Factor graph based simultaneous localization and mapping using multipath channel information," in IEEE International Conference on Communications Workshops (ICC Workshops), May 2017, pp. 652-658.

[10] E. Leitinger, S. Grebien, and K. Witrisal, "Multipath-Based SLAM Exploiting AoA and Amplitude Information," in IEEE International Conference on Communications Workshops (ICC Workshops), May 2019, pp. 1-7.

[11] H. Wymeersch, N. Garcia, H. Kim, G. Seco-Granados, S. Kim, F. Wen, and M. Fröhle, "5G mm Wave Downlink Vehicular Positioning," in IEEE Global Communications Conference (GLOBECOM), Dec. 2018 , pp. 206-212.

[12] H. Kim, H. Wymeersch, N. Garcia, G. Seco-Granados, and S. Kim, "5G mmWave Vehicular Tracking," in 52nd Asilomar Conference on Signals, Systems, and Computers, Oct. 2018, pp. 541-547.
[13] S. Kay, Fundamentals of Statistical Signal Processing: Estimation Theory, ser. Fundamentals of Statistical Signal Processing. PrenticeHall PTR, 1998

[14] T. Bailey and H. Durrant-Whyte, "Simultaneous localization and mapping (SLAM): part II," IEEE Robot. Autom. Mag., vol. 13, no. 3, pp. 108-117, Sep. 2006.

[15] M. Ulmschneider, C. Gentner, T. Jost, and A. Dammann, "Association of Transmitters in Multipath-Assisted Positioning," in IEEE Global Communications Conference (GLOBECOM), Singapore, Dec. 2017.

[16] — , "Multiple Hypothesis Data Association for Multipath-Assisted Positioning," in 14th Workshop on Positioning, Navigation and Communications (WPNC), Oct. 2017.

[17] S. Thrun, M. Montemerlo, D. Koller, B. Wegbreit, J. Nieto, and E. Nebot, "FastSLAM: An Efficient Solution to the Simultaneous Localization And Mapping Problem with Unknown Data Association,' Journal of Machine Learning Research, vol. 4, no. 3, pp. 380-407, 2004.

[18] M. Ulmschneider and C. Gentner, "Improving Maps of Physical and Virtual Radio Transmitters," in ION GNSS+, Sep. 2018.

[19] D. Tse and P. Viswanath, Fundamentals of Wireless Communication. Cambridge University Press, 2005.

[20] T. Jost, W. Wang, U. Fiebig, and F. Perez-Fontan, "Detection and Tracking of Mobile Propagation Channel Paths," IEEE Trans. Antennas Propag., vol. 60, no. 10, pp. 4875-4883, Oct. 2012.

[21] B. Fleury, M. Tschudin, R. Heddergott, D. Dahlhaus, and K. Pedersen, "Channel Parameter Estimation in Mobile Radio Environments Using the SAGE Algorithm," IEEE J. Sel. Areas Commun., vol. 17, no. 3, pp 434-450, Mar. 1999

[22] A. P. Dempster, N. M. Laird, and D. B. Rubin, "Maximum Likelihood from Incomplete Data via the EM Algorithm," Journal of the Royal Statistical Society. Series B (Methodological), vol. 39, no. 1, pp. 1-38, 1977.

[23] A. Doucet, N. de Freitas, K. Murphy, and S. Russell, "Rao-blackwellised Particle Filtering for Dynamic Bayesian Networks," in Proceedings of the Sixteenth Conference on Uncertainty in Artificial Intelligence, ser UAI'00. San Francisco, CA, USA: Morgan Kaufmann Publishers Inc., 2000, pp. 176-183.

[24] M. Arulampalam, S. Maskell, N. Gordon, and T. Clapp, "A Tutorial on Particle Filters for Online Nonlinear/non-Gaussian Bayesian Tracking,' IEEE Trans. Signal Process., vol. 50, no. 2, pp. 174-188, Feb. 2002.

[25] C. Gentner, R. Pöhlmann, M. Ulmschneider, T. Jost, and S. Zhang, "Positioning Using Terrestrial Multipath Signals and Inertial Sensors," Mobile Information Systems, vol. 2017, 2017.

[26] S. Thrun, W. Burgard, and D. Fox, Probabilistic Robotics, ser. Intelligent robotics and autonomous agents. MIT Press, 2005 